\title{
Front Matter: Volume 9414
}

, "Front Matter: Volume 9414," Proc. SPIE 9414, Medical Imaging 2015:

Computer-Aided Diagnosis, 941401 (11 May 2015); doi: 10.1117/12.2194210

SPIE. Event: SPIE Medical Imaging, 2015, Orlando, Florida, United States 


\title{
PROGRESS IN BIOMEDICAL OPTICS AND IMAGING
}

\section{Medical Imaging 2015: Computer-Aided Diagnosis}

\author{
Lubomir M. Hadjiiski \\ Georgia D. Tourassi \\ Editors
}

22-25 February 2015
Orlando, Florida, United States

Sponsored by

SPIE

Cosponsored by

ALIO Industries (United States) - Alpinion Medical Systems (United States) • Modus Medical Devices Inc. (Canada) • Bruker (United States)

Cooperating Organizations

AAPM-American Association of Physicists in Medicine (United States) - APS—American Physiological Society (United States) • CARS - Computer Assisted Radiology and Surgery (Germany) • Medical Image Perception Society (United States) • Radiological Society of North America (United States) - Society for Imaging Informatics in Medicine (United States) World Molecular Imaging Society • The DICOM Standards Committee

Published by

SPIE 
The papers included in this volume were part of the technical conference cited on the cover and title page. Papers were selected and subject to review by the editors and conference program committee. Some conference presentations may not be available for publication. The papers published in these proceedings reflect the work and thoughts of the authors and are published here in as submitted. The publisher is not responsible for the validity of the information or for any outc omes resulting from reliance thereon.

Please use the following format to cite material from this book:

Author(s), "Title of Paper," in Medical Imaging 2015: Computer-Aided Diagnosis, edited by Lubomir M. Hadjiiski, G eorgia D. Toura ssi, Proc eedings of SPIE Vol. 9414 (SPIE, Bellingham, WA, 2015) Article CID Number.

ISSN: $1605-7422$

ISBN: 9781628415049

Published by

SPIE

P.O. Box 10, Bellingham, Washington 98227-0010 USA

Telephone +1 3606763290 (Pacific Time)· Fax +1 3606471445

SPIE.org

Copyright @2015, Soc iety of Photo-O ptic al Instrumentation Engineers.

Copying of material in this book for intemal or personal use, or for the intemal or personal use of specific clients, beyond the fa ir use provisions granted by the U.S. Copyright Law is authorized by SPIE subject to payment of copying fees. The Transactional Reporting Service base fee for this volume is $\$ 18.00$ per article (or portion thereof), which should be paid directly to the Copyright Clearance Center (CCC), 222 Rosewood Drive, Danvers, MA 01923. Payment may also be made electronically through CCC Online at copyright.com. Other copying for republication, resale, advertising or promotion, or any form of systematic or multiple reproduction of a ny material in this book is prohibited except with permission in writing from the publisher. The CCC fee code is $1605-7422 / 15 / \$ 18.00$.

Printed in the United States of America.

Public ation of record for individual papers is online in the SPIE Dig ital Library.

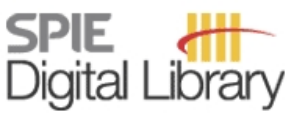

SPIEDigitalLibrary.org

Paper Numbering: Proceedings of SPIE follow an e-First publication model, with papers published first online and then in print. Papers are published as they are submitted and meet publication criteria. A unique citation identifier (CID) number is assigned to each article at the time of the first publication. Utilization of CIDs allows articles to be fully citable as soon as they are published online, and connects the same identifier to all online, print, and electronic versions of the publication. SPIE uses a six-dig it CID article numbering system in which:

- The first four digits correspond to the SPIE volume number.

- The last two digits indicate publication order within the volume using a Base 36 numbering

system employing both numerals and letters. These two-number sets start with $00,01,02,03,04$, 05, 06, 07, 08, 09, 0A, 0B ... 0Z, followed by 10-1Z, 20-2Z, etc.

The CID Number appears on each page of the manuscript. The complete citation is used on the first page, and an abbreviated version on subsequent pages. 


\title{
Contents
}

\author{
xiii Authors \\ xix Conference Committee \\ xxiii Introduction \\ xxvii 2015 Medical Imaging Paper Award Recipients
}

\section{Part One}

\section{MUSCULOSKELETAL AND MISCELLANEOUS}

941402 Automatic diagnosis of inflammatory muscle disease for MRI using computer-extracted features of bivariate histograms [9414-1]

941403 Segmentation of the sternum from low-dose chest CT images [9414-2]

941404 Detection of degenerative change in lateral projection cervical spine x-ray images [9414-3]

941405 Diagnostic index of 3D osteoarthritic changes in TMJ condylar morphology [9414-4]

941406 3D statistical shape models incorporating 3D random forest regression voting for robust CT liver segmentation [9414-5]

\section{LUNG AND CHEST I}

941407 Pulmonary embolism detection using localized vessel-based features in dual energy CT [9414-6]

941408 Robustness evaluation of a computer-aided detection system for pulmonary embolism (PE) in CTPA using independent test set from multiple institutions [9414-7]

941409 Automatic detection of spiculation of pulmonary nodules in computed tomography images [9414-8]

9414 OA Improving CAD performance by seamless insertion of pulmonary nodules in chest CT exams [9414-9]

9414 OB Analysis of the Vancouver lung nodule malignancy model with respect to manual and automated segmentation [9414-10]

9414 OC Factors affecting uncertainty in lung nodule volume estimation with CT: comparisons of findings from two estimation methods in a phantom study [9414-1 1] 
9414 OD Automatic machine learning based prediction of cardiovascular events in lung cancer screening data [9414-12]

9414 OE Automatic selection of best quality vessels from multiple-phase coronary CT angiography (cCTA) [9414-13]

9414 OF Determining degree of optic nerve edema from color fundus photography [9414-14]

9414 OG Automated segmentation of cardiac visceral fat in low-dose non-contrast chest CT images [9414-15]

$9414 \mathrm{OH}$ Development of a screening tool for staging of diabetic retinopathy in fundus images [9414-16]

BREAST I

9414 Ol Segmentation of the whole breast from low-dose chest CT images [9414-17]

$94140 \mathrm{~J}$ Vessel segmentation in screening mammograms [9414-18]

9414 OK Comparison of computer-aided detection of clustered microcalcifications in digital mammography and digital breast tomosynthesis [9414-19]

$9414 \mathrm{OL} \quad$ Initial experience with computer aided detection for microcalcification in digital breast tomosynthesis [9414-42]

9414 OM Signal enhancement ratio (SER) quantified from breast DCE-MRI and breast cancer risk [9414-21]

9414 ON A comparative analysis of 2D and 3D CAD for calcifications in digital breast tomosynthesis [9414-22]

941400 A superpixel-based framework for automatic tumor segmentation on breast DCE-MRI [9414-23]

\section{PROSTATE AND COLON I}

9414 OP Connection method of separated luminal regions of intestine from CT volumes [9414-24]

$94140 Q$ Electronic cleansing for dual-energy CT colonography based on material decomposition and virtual monochromatic imaging [9414-25]

9414 OR Distance weighted 'inside disc' classifier for computer-aided diagnosis of colonic polyps [9414-26] 
9414 OS Efficient Hilbert transform-based alternative to Tofts physiological models for representing MRI dynamic contrast-enhanced images in computer-aided diagnosis of prostate cancer [9414-27]

9414 OT The evaluation of multi-structure multi-atlas pelvic anatomy features in a prostate MR lymphography CAD system [9414-28]

\section{KEYNOTE AND NOVEL METHODS}

9414 OV Deep learning with non-medical training used for chest pathology identification [9414-30]

9414 OW Texture classification of anatomical structures in CT using a context-free machine learning approach [9414-31]

$94140 X \quad$ Multi-test cervical cancer diagnosis with missing data estimation [9414-32]

HEAD AND NECK

9414 OY Atlas-based segmentation of brainstem regions in neuromelanin-sensitive magnetic resonance images [9414-33]

$94140 Z$ Automatic anatomy recognition in post-tonsillectomy MR images of obese children with OSAS [9414-34]

941410 Multi-fractal detrended texture feature for brain tumor classification [9414-35]

941411 Small white matter lesion detection in cerebral small vessel disease [9414-36]

941412 Automated prediction of tissue outcome after acute ischemic stroke in computed tomography perfusion images [9414-37]

941413 Automated segmentation of thyroid gland on CT images with multi-atlas label fusion and random classification forest [9414-38]

941414 Detection of Alzheimer's disease using group lasso SVM-based region selection [9414-39]

BREAST II

941415 Identifying metastatic breast tumors using textural kinetic features of a contrast based habitat in DCE-MRI [9414-40]

941416 Association of mammographic image feature change and an increasing risk trend of developing breast cancer: an assessment [9414-76]

941417 Local mammographic density as a predictor of breast cancer [9414-20]

941418 Digital breast tomosynthesis: application of 2D digital mammography CAD to detection of microcalcification clusters on planar projection image [9414-43] 
941419 Combination of conspicuity improved synthetic mammograms and digital breast tomosynthesis: a promising approach for mass detection [9414-44]

PROSTATE AND COLON II

9414 1A Characterization of aggressive prostate cancer using ultrasound RF time series [9414-106]

$94141 \mathrm{~B} \quad$ Multimodal classification of prostate tissue: a feasibility study on combining multiparametric MRI and ultrasound [9414-46]

$94141 \mathrm{C}$ Towards intraoperative surgical margin assessment and visualization using bioimpedance properties of the tissue [9414-47]

9414 1D Quantification, validation, and follow-up of small bowel motility in Crohn's disease [9414-48]

$94141 \mathrm{E} \quad$ A content-based image retrieval method for optical colonoscopy images based on image recognition techniques [9414-49]

9414 IF Detection of colonic polyp candidates with level set-based thickness mapping over the colon wall [9414-50]

VESSELS, HEART, AND EYE II

$94141 \mathrm{G}$ Automated measurement of pulmonary artery in low-dose non-contrast chest CT images [9414-51]

$94141 \mathrm{H} \quad$ Quantitative analysis of arterial flow properties for detection of non-calcified plaques in ECG-gated coronary CT angiography [9414-52]

$941411 \quad$ Automated age-related macular degeneration classification in OCT using unsupervised feature learning [9414-53]

$94141 \mathrm{~J} \quad$ Automatic discrimination of color retinal images using the bag of words approach (First Place RFW All Conference Best Student Paper Award) [9414-54]

\section{LUNG AND CHEST II}

9414 1K Reducing annotation cost and uncertainty in computer-aided diagnosis through selective iterative classification [9414-55]

$94141 \mathrm{~L}$ A computer-aided diagnosis system to identify regions of pathologic change in temporal subtraction images of the chest [9414-56]

$94141 \mathrm{M} \quad$ Exploring new quantitative $\mathrm{CT}$ image features to improve assessment of lung cancer prognosis [9414-57] 
$94141 \mathrm{~N} \quad$ Nonlinear dimensionality reduction of CT histogram based feature space for predicting recurrence-free survival in non-small-cell lung cancer [9414-58]

941410 Computer-aided detection of lung cancer: combining pulmonary nodule detection systems with a tumor risk prediction model [9414-59]

\section{Part Two}

\section{MULTI-ORGAN}

$94141 \mathrm{P} \quad$ Pneumothorax detection in chest radiographs using local and global texture signatures [9414-60]

$94141 Q \quad$ Computer-aided detection of bladder mass within contrast-enhanced region of CTU [9414-61]

$94141 R \quad$ Automatic identification of IASLC-defined mediastinal lymph node stations on CT scans using multi-atlas organ segmentation [9414-62]

$941415 \quad$ Ureter segmentation in CT urography (CTU) by COMPASS with multiscale Hessian enhancement [9414-63]

$94141 \mathrm{U}$ Prediction of treatment outcome in soft tissue sarcoma based on radiologically defined habitats [9414-65]

POSTERS: BREAST

$94141 \mathrm{~V}$ Potential reasons for differences in CAD effectiveness evaluated using laboratory and clinical studies [9414-41]

$94141 \mathrm{~W} \quad$ Usefulness of histogram analysis of spatial frequency components for exploring the similarity and bilateral asymmetry in mammograms [9414-66]

$94141 \mathrm{X} \quad$ Automated lesion detection in dynamic contrast enhanced magnetic resonance imaging of breast [9414-67]

$94141 Y \quad$ Fully automated quantitative analysis of breast cancer risk in DCE-MR images [9414-68]

941412 Quantification of tumor changes during neoadjuvant chemotherapy with longitudinal breast DCE-MRI registration [9414-69]

941420 A new Fourier transform based CBIR scheme for mammographic mass classification: a preliminary invariance assessment [9414-70]

$941421 \quad$ Utilizing digital breast tomosynthesis projection views correlation for microcalcification enhancement for detection purposes [9414-71]

941422 A new breast cancer risk analysis approach using features extracted from multiple subregions on bilateral mammograms [9414-72] 
941423 Chest wall segmentation in automated 3D breast ultrasound using rib shadow enhancement and multi-plane cumulative probability enhanced map [9414-73]

941424 A new CAD approach for improving efficacy of cancer screening (Cum Laude Poster Award) [9414-74]

941425 Feature extraction from inter-view similarity of DBT projection views [9414-75]

941426 Simplified false-positive reduction in computer-aided detection scheme of clustered microcalcifications in digital breast tomosynthesis [9414-77]

941428 Automatic breast density classification using a convolutional neural network architecture search procedure [9414-79]

941429 Automated detection of breast tumor in MRI and comparison of kinetic features for assessing tumor response to chemotherapy [9414-80]

$94142 \mathrm{~A}$ Preliminary study on the automated detection of breast tumors using the characteristic features from unenhanced MR images [9414-81]

$94142 B \quad$ Estimation of corresponding locations in ipsilateral mammograms: a comparison of different methods [9414-82]

POSTERS: HEAD AND NECK

9414 2D 3D active shape models of human brain structures: application to patient-specific mesh generation [9414-84]

$94142 \mathrm{E}$ Computer-aided recognition of dental implants in x-ray images [9414-85]

$94142 \mathrm{~F}$ Computer aided detection of brain micro-bleeds in traumatic brain injury [9414-87]

$94142 \mathrm{G}$ Automatic segmentation method of striatum regions in quantitative susceptibility mapping images [9414-88]

$94142 \mathrm{H} \quad$ Decoding brain cancer dynamics: a quantitative histogram-based approach using temporal MRI [9414-89]

941421 Discriminative analysis of non-linear brain connectivity for leukoaraiosis with resting-state fMRI [9414-90]

$94142 \mathrm{~J} \quad$ Automated classification of mandibular cortical bone on dental panoramic radiographs for early detection of osteoporosis [9414-91]

$94142 \mathrm{~K}$ Imbalanced learning for clinical survival group prediction of brain tumor patients [9414-92]

$94142 \mathrm{~L} \quad$ Longitudinal MRI assessment: the identification of relevant features in the development of Posterior Fossa Syndrome in children [9414-93]

$94142 \mathrm{M}$ Quantification of vocal fold motion using echography: application to recurrent nerve paralysis detection [9414-94] 
$94142 \mathrm{~N}$ Automatic detection of larynx cancer from contrast-enhanced magnetic resonance images [9414-95]

POSTERS: LUNG AND CHEST

941420 Quantitative assessment of smoking-induced emphysema progression in longitudinal CT screening for lung cancer [9414-96]

$94142 \mathrm{P} \quad$ A novel spherical shell filter for reducing false positives in automatic detection of pulmonary nodules in thoracic CT scans [9414-97]

$94142 Q \quad$ An outlier filtering approach for machine sourced weak segmentations [9414-98]

$94142 R$ Segmentation of interstitial lung disease patterns in HRCT images [9414-99]

$94142 S$ Semi-automated segmentation of solid and GGO nodules in lung CT images using vessellikelihood derived from local foreground structure [9414-100]

9414 2T Improved pulmonary nodule classification utilizing lung parenchyma texture features [9414-101]

$94142 \mathrm{U}$ Quantification of pulmonary vessel diameter in low-dose CT images [9414-102]

9414 2V Automated detection of lung tumors in PET/CT images using active contour filter [9414-103]

9414 2W Peripleural lung disease detection based on multi-slice CT images [9414-104]

POSTERS: PROSTATE AND COLON

$94142 \mathrm{X}$ Prostate cancer detection from model-free I1-weighted time series and diffusion imaging [9414-45]

$94142 Y \quad$ Context-specific method for detection of soft-tissue lesions in non-cathartic low-dose dualenergy CT colonography [9414-105]

POSTERS: VESSELS, HEART, AND EYE

941430 Automated detection of Schlemm's canal in spectral-domain optical coherence tomography [9414-108]

941431 Computer-based assessment of right ventricular regional ejection fraction in patients with repaired Tetralogy of Fallot [9414-109]

941432 Automatic generation of endocardial surface meshes with 1-to-1 correspondence from cine-MR images [9414-110] 
941433 Semi-automated measurements of heart-to-mediastinum ratio on 123]-MIBG myocardial scintigrams by using image fusion method with chest $x$-ray images [9414-111]

941434 Myocardial strain estimation from CT: towards computer-aided diagnosis on infarction identification [9414-112]

941435 Heart rate measurement based on face video sequence [9414-113]

941438 Characterization of vascular tree architecture using the Tokunaga taxonomy [9414-116]

POSTERS: MUSCULOSKELETAL AND MISCELLANEOUS

941439 Bone age assessment meets SIFT [9414-117]

9414 3A A novel framework for the temporal analysis of bone mineral density in metastatic lesions using CT images of the femur [9414-118]

$94143 \mathrm{~B} \quad$ Spinal focal lesion detection in multiple myeloma using multimodal image features [9414-119]

$94143 C$ Knee osteoarthritis image registration: data from the Osteoarthritis Initiative [9414-120]

$94143 D$ Dynamic cortex stripping for vertebra evaluation [9414-121]

9414 3E Prognosis of intervertebral disc loss from diagnosis of degenerative disc disease [9414-122]

9414 3F A web-based procedure for liver segmentation in CT images [9414-123]

\section{POSTERS: MULTI-ORGAN}

$94143 G \quad$ Evaluation of chemotherapy response in ovarian cancer treatment using quantitative CT image biomarkers: a preliminary study [9414-124]

$94143 \mathrm{H} \quad$ Voxel-based registration of simulated and real patient CBCT data for accurate dental implant pose estimation [9414-125]

$941431 \quad$ Automated classification of bone marrow cells in microscopic images for diagnosis of leukemia: a comparison of two classification schemes with respect to the segmentation quality [9414-126]

9414 3J Human wound photogrammetry with low-cost hardware based on automatic calibration of geometry and color [9414-127]

$94143 \mathrm{~K} \quad$ Automatic anatomy partitioning of the torso region on $\mathrm{CT}$ images by using multiple organ localizations with a group-wise calibration technique [9414-128]

9414 3L Automated torso organ segmentation from 3D CT images using structured perceptron and dual decomposition [9414-129] 
$94143 \mathrm{M} \quad$ Automatic detection and segmentation of vascular structures in dermoscopy images using a novel vesselness measure based on pixel redness and tubularness [9414-130]

$94143 \mathrm{~N}$ Investigation of optimal feature value set in false positive reduction process for automated abdominal lymph node detection method [9414-131]

941430 A pilot study on bladder wall thickness at different filling stages [9414-132] 
Proc. of SPIE Vol. $9414941401-12$

Downloaded From: https://www.spiedigitallibrary.org/conference-proceedings-of-spie on 26 Apr 2023 Terms of Use: https://www.spiedigitallibrary.org/terms-of-use 


\section{Authors}

Numbers in the index correspond to the last two digits of the six-digit citation identifier (CID) article numbering system used in Proceedings of SPIE. The first four digits reflect the volume number. Base 36 numbering is employed for the last two digits and indicates the order of articles within the volume. Numbers start with 00, 01, 02, 03, 04, 05, 06, 07, 08, 09, 0A, 0B...0Z, followed by 10-1Z, 20-2Z, etc.

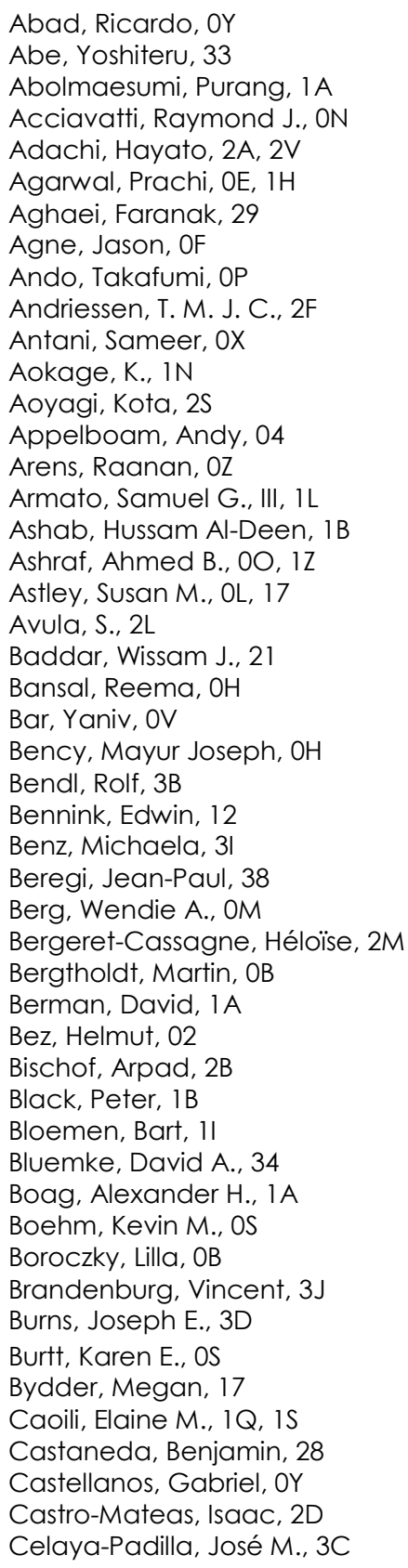

Cerrolaza, Juan J., 1D

Cevidanes, Lucia, 05

Cha, Kenny, 1Q, IS

Chae, Eun Young, 26

Chae, Seung-Hoon, 26

Chan, Heang-Ping, 08, 0E, OK, 18, 1H, 1Q, is

Chang, Kevin, 13

Chang, Silvia D., 2X

Chaudhury, Baishali, 15, $1 \mathrm{U}$

Chen, Huayue, 3K

Chen, Marcus, 34

Chen, Weijie, OA

Chen, Xin, 17

Chen, Xing, 35

Choi, Young-Wook, 26

Choyke, Peter, OS

Chughtai, Aamer, 08, 0E, 1H

Ciompi, Francesco, 09, 10

Cohan, Richard H., 1Q, IS

Cohen, Mike-Ely, 2M

Conant, Emily F., ON, 0O, $1 \mathrm{Z}$

Conklin, Laurie, ID

Correia, André Ricardo, $3 \mathrm{H}$

Dankbaar, Jan Willem, 12

Dash, Jatindra Kumar, 2R

Davatzikos, Christos, 00, $1 \mathrm{Z}$

Debats, O., OT

de Jong, Hugo, 12

de Jong, Pim A., OD

de Leeuw, Frank Erik, 11

DeMichele, Angela, 12

Depeursinge, Adrien, 07, 0W

Derikx, Loes C., 3A

Deserno, Thomas M., 30, 39, 3J

de Vos, Bob D., OD

Dhara, Ashis Kumar, $\mathrm{OH}$

Diamant, Idit, OV

Dicente Cid, Yashin, 07

Dilger, S. K., 2T

Ding, Kai, $3 G$

Dirksen, Asger, 10

Dorssers, Frank, 2P

Doshi, Trushali, 2N

Drukteinis, Jennifer S., 15

Duan, Chaijie, IF

Duque, Duarte, 2E

Edirisinghe, Eran, 02

Eguchi, K., 1N, 2O, 2W

Ehrhardt, Jan, 2B 
Ehteshami Bejnordi, Babak, 2P

Emaminejad, Nastaran, 1M

Evans, Gareth D., 17

Fairbanks, Duncan, 15

Fan, Yong, 14

Farhidzadeh, Hamidreza, $1 \mathrm{U}$

Fernandes, Valter, 3H

Fernández-Seara, María A., OY

Ferreira, Adriano, 2E

Ferreira, Ernesto, 2E

Ferrer, Jose, 28

Foncubierta-Rodríguez, Antonio, 07, 0W

Fonseca, Jaime C., 3H

Fonseca, Pablo, 28

Frangi, Alejandro F., 2D

Fränzle, Andrea, 3B

Frazer, Helen, $1 X$

Frouin, Frédérique, $2 \mathrm{M}$

Fujita, Hiroshi, 2A, 2J, 2V, 33, 3K

Fujiwara, Michitaka, 3N

Fukui, Tatsumasa, 2J

Furst, Jacob, 1K, 2Q

Furukawa, Kazuhiro, OP, 3N

Gadde, Soujanya, 17

Galarreta-Valverde, Miguel A., 38

Galván-Tejada, Jorge I., 3C

Gaonkar, Bilwaj, 00

Garvin, Mona K., OF

Gatenby, Robert A., 15, 1U, 2H, 2K

Gavrielides, Marios A., OC

Geva, Ofer, 1P

Ghafoorian, Mohsen, 11, 2F

Gilbert, F. J., OL

Gillies, Robert J., 15, 1U, 2H, 2K

Goldenberg, S. Larry, 1B, 2X

Goldgof, Dmitry B., 15, 1U, 2H, 2K

Gomes, Liliane R., 05

Gomes, Marcelo, 05

Gonçalves, João Roberto, 05

Goraj, B. M., 2F

Goto, Hidemi, OP, 3N

Greenspan, Hayit, OV, IP

Grose, Derek, 2N

Gu, Yajia, $1 Y$

Guan, Yubao, 1M

Guerrero, Jorge, 28

Gundreddy, Rohith Reddy, 20

Gupta, Amod, $\mathrm{OH}$

Gur, David, OM

Haak, Daniel, 39, 3J

Hachi, Siham, 2M

Hadjiiski, Lubomir M., 08, 0E, OK, 18, 1H, 1Q, is

Haferlach, Torsten, 31

Hall, Lawrence O., 15, 1U, 2H, 2K

Halter, Ryan, $1 \mathrm{C}$

Hammond, E., 2T

Han, Hao, $1 \mathrm{~F}$

Handels, Heinz, 2B

Haq, Nandinee Fariah, 1B, 2X

Haq, R., OL
Hara, Takeshi, 2J, 33, 3K

Harkness, Elaine F., OL, 17

Hayashi, Tatsuro, 2J

Hayashi, Yuichiro, 3L

$\mathrm{He}$, Xin, IV

Helvie, Mark, OK, 18

Henschke, Claudia I., 0G, 0I, IG

Heskes, Tom, 11

Hillengass, Jens, 3B

Hirai, Toshinori, $2 \mathrm{G}$

Hirooka, Yoshiki, OP

Ho, Charles, 1L

Hoffman, Joanne, $1 R$

Hong, Helen, 23

Horiba, Kazuki, 2J

Hoshi, Hiroaki, 3K

Hoshmand, Vahid, 12

Howell, Anthony, 17

Hoyng, Carel, 11

Hu, Xiaoxin, IY

$\mathrm{Hu}$, Yifan, OR

Huang, Xiaolei, OX

Huisman, H., OT

Hutchinson, Charles, 02

Hyams, Elias S., 1C

Iftekharuddin, Khan M., 10

Imani, Farhad, IA

Išgum, Ivana, OD

Ishihara, Tadahiko, 33

Ito, Masaaki, 3N

Jack, James, 02

Jackowski, Marcel P., 38

Jacobs, Colin, 09, 10

Jankowitz, Rachel C., OM

Jebri, Beyrem, 04

Jeong, Ji-Wook, 26

Jiang, Luan, $1 Y$

Jiang, YunQing, 00

Jiménez del Toro, Oscar Alfonso, OW

Jonas, Stephan, 39, 3J

Jones, Edward C., 1B, 2X

Jones, Terence, 02

Jose, Abin, $3 \mathrm{~J}$

Judisch, A., 2T

Jung, Bryan, 05

Kaneko, M., 1N, 2O, 2W

Kanematsu, Masayuki, 3K

Kang, Yan, 1M, 24

Kardon, Randy H., OF

Karssemeijer, Nico, 0J, 11

Kashif, Muhammad, 39

Katafuchi, Tetsuro, 33

Katsumata, Akitoshi, 2J

Kawai, Ryosuke, 33

Kawata, Y., 1N, 2O, 2W

Kazerooni, Ella A., 08, 0E, 1H

Keller, Brad M., ON, 00, 12

Khan, Shadab, 1C

Khandelwal, Niranjan, 2R

Kharazmi, Pegah, 3M 
Khojaste, Amir, 1A

Kim, Dae Hoe, 19, 21, 25

Kim, Edward, OX

Kim, Eun Joon, 21

Kim, Hak Hee, 26

Kim, Hannah, 23

Kim, Hyeonjin, 23

Kim, Lauren, 13, 1R

Kim, Se Hyung, $0 Q$

Kim, Seong Tae, 19, 25

Kitasaka, Takayuki, OP, 3L, 3N

Klinder, Tobias, OB

Knapp, Karen, 04

Knoop, Tom H., 3A

Kobayashi, Toshiki, 2V

Konen, Eli, 1P

Kontos, Despina, 00, 12

Kotagiri, Romamohanarao, $1 \mathrm{X}$

Kozlowski, Piotr, 1B, 2X

Krappe, Sebastian, 31

Krüger, Julia, 2B

Kumar, Prafulla, 2R

Kuriakose, Jean W., 08, OE, $1 \mathrm{H}$

Kurland, Brenda F., OM

Kusumoto, M., 1N, 2O, 2W

Lai, Youzhi, 2I

Lazard, Diane, 2M

Leader, Joseph K., 16

Lee, Katherine, $1 \mathrm{~L}$

Lee, Sooyeul, 26

Lee, Tim K., 3M

Leenhardt, Laurence, $2 \mathrm{M}$

Lefort, Muriel, 2M

Lelieveldt, Boudewijn P. F., 14

Lewis, E., $2 \mathrm{~L}$

Li, Ang, 2M

Li, Baojuan, 30

Li, Lihong, 1F

Li, Lihua, 24

Li, Qiang, 1Y

Li, Qin, OC

Li, S., 3E

Liang, Mingzhu, OG, IG

Liang, Xi, $1 \mathrm{X}$

Liang, Zhengrong, $\mathrm{OR}, 1 \mathrm{~F}, 3 \mathrm{O}$

Lieberman, Sivan, $1 \mathrm{P}$

Lim, C. W., 32

Lim, Yi† Y., OL, 17

Lin, A., 3E

Linguraru, Marius George, 1D

Liu, Hong, 16, 3G

Liv, Jiamin, 13, 1R

Liu, Jordan, OE

Liu, Shuang, 03, 01

Liv, Yang, 30

Long, L. Rodney, OX

Lu, Hongbing, 30

Lu, Le, 13

Lu, Yao, OK, 18

Lui, Harvey, 3M
Luo, Ming, 3F

Lure, Fleming, 1M, 24

MacKenzie, Kenneth, 2N

Madhavi, Vaddepalli, 2R

Mahara, Aditya, 1C

Maidment, Andrew D. A., ON

Maier-Hein, Klaus H., 06

Marchiori, Elena, 11

Margolin, Elza, 2Q

Marx, Mirko, 2B

Matsuhiro, M., 2O, 2W

Matsumoto, Sumiaki, $2 S$

Matsunobu, Yusuke, IW

Maxwell, Anthony J., OL, 17

Mays, Randall, 10

McMeekin, Scott, $3 G$

Meijs, M., OT

Meinzer, Hans-Peter, 06

Mekkaoui, Choukri, 38

Mendoza, Julio, 28

Menegaux, Fabrice, 2M

Meriaudeau, F., $1 \mathrm{~J}$

Misawa, Kazunari, 3N

Miyajo, Satomi, 2A

Mizuguchi, R., 20

Mizuno, Shinji, 3N

Moore, Kathleen, $3 G$

Moradi, Mehdi, 1A, 1B, 2X

Morais, Pedro, 2E, 3H

Mordang, J. J., OJ

Moreira, António H. J., 2E, 3H

Mori, Kensaku, OP, 3L, 3N

Morishita, Junji, IW

Morita, Syoichi, 3K

Moriyama, N., 20

Moschidis, Emmanouil, 17

Mousavi, Parvin, IA

Mukhopadhyay, Sudipta, $\mathrm{OH}, 2 \mathrm{R}$

Müller, Henning, 07, OW

Muñoz-Barrutia, Arrate, OY, $2 \mathrm{U}$

Münzenmayer, Christian, 3l

Murakawa, Masahiro, 1E

Murakawa, Saki, 2G

Muramatsu, Chisako, 2J, 33

Myers, Kyle J., OC

Nakamura, Yoshihiko, 3N

Nakano, Y., 2O, 2W

Näppi, Janne J., $0 Q, 2 Y$

Nawano, Shigeru, 3N

Newell, J. D., Jr., 2T

Niki, N., Jr., 1N, 2O, 2W

Nimura, Yukitaka, 3L, 3N

Nir, Guy, 1B

Nishio, Masami, 2A, 2V

Norajitra, Tobias, 06

Nosato, Hirokazu, $1 \mathrm{E}$

Oda, Masahiro, OP

Odhner, Dewey, $0 Z$

Ohmatsu, H., 1N, 2O, 2W

Ohmi, Kumiko, 2A 
Ohno, Yoshiharu, 2S

Okazaki, Tomoya, 2S

Ortiz-de-Solórzano, Carlos, OY, $2 \mathrm{U}$

Osman, Said, 3E

Otsuka, Mayu, 17

Ou, Yangming, $1 \mathrm{Z}$

Paniagua, Beatriz, 05

Pastor, María A., OY

Pastor, Pau, OY

Patel, Smita, 08, 1H

Peng, Jennifer Q., ID

Pereira, Fabricio, 38

Petrick, Nicholas, OA, OC, OS

Petropoulakis, Lykourgos, 2N

Pezeshk, Aria, OA

Philipsen, Rick, 11

Phillips, Michael, 04

Pickhardt, Perry J., OR

Pinho, A. C. M., 3H

Pinto, Joseph, 28

Pinto, Peter, OS

Platel, Bram, 11, 2F

Platon, Alexandra, 07

Poletti, Pierre-Alexandre, 07

Pozo, Jose M., 2D

Prokop, M., 09

Pu, Jiantao, 24

Puigvert, Marc, OY

Qian, Wei, 1M, 22, 24

Queirós, Sandro, 2E, 3H

Qui, Yuchen, 20, 24, $3 \mathrm{G}$

Raghavan, Meera, $1 \mathrm{U}$

Raicu, Daniela, 1K, 2Q

Ramakrishnan, Vignesh, 30

Rangayyan, Rangaraj M., $\mathrm{OH}$

Ravikumar, Nishant, 2D

Ray, Shonket, ON

Reeves, Anthony P., 03, 0G, 0I, IG

Regge, Daniele, $2 Y$

Reuben, Adam, 04

Reza, Syed M. S., 10

Riely, Amelia, $1 \mathrm{~K}$

Ro, Yong Man, 19, 21, 25

Rodrigues, Nuno F., 2E, 3H

Romano, W., 3E

Rosen, Mark, $1 \mathrm{Z}$

Rudyanto, Rina D., $2 U$

Ruellas, Antonio C., 05

Russ, Gilles, 2M

Russo, Robin, $2 \mathrm{H}$

Sablan, Kyle, $1 \mathrm{~K}$

Sadek, I., $1 \mathrm{~J}$

Safdar, Nabile, 1D

Sahiner, Berkman, OA, OC, OS, IV

Sakanashi, Hidenori, IE

Salcudean, Septimiu E., 1B

Salvatore, Mary, ol

Samala, Ravi K., OK, 18

Samuelson, Frank, IV

Sánchez, Clara I., 11
Saverberi, Eric E., IA

Schned, Alan, 1C

Scholten, E. Th., 09

Setio, Arnaud A. A., 10

Shiotsuki, Kenshi, IW

Sidibé, D., $1 \mathrm{~J}$

Siemens, D. Robert, 1A

Sieren, J. C., 2T

Sin, Sanghun, $0 Z$

Slabaugh, Greg, 04

Slump, Cornelis H., 3A

Song, Bowen, OR

Soraghan, John, 2N

Spiteri, M., 2L

Stavrinos, Paula, 17

Stieger, James, 3D

Stoecker, William V., 3M

Styner, Martin A., 05

Su, Y., 31, 32

Sumkin, Jules, OM

Summers, Ronald M., OS, 13, 1R, 34, 3D

Sun, Wenqing, 22

Sun, Zhuo, 14

Suzuki, H., 2O, 2W

Sze, Raymond, 1D

Tachibana, Rie, $\mathrm{OQ}$

Takahashi, Eiichi, 1E

Takahashi, Katsuaki, 2V

Takeguchi, Tomoyuki, $2 S$

Tamaki, Tsuneo, 2V

Tamez-Peña, José G., 3C

Tan, M. L., 31

Tan, Maxine, 16, 20, 24, 29, $3 \mathrm{G}$

Tan, R.-S., 31, 32

Tate, C., OL

Tay, K., 3E

Taylor, Zeike A., 2D

Tee, Michael, 34

Teo, S.-K., 31, 32

Teramoto, Atsushi, 2A, 2V

ter Haar Romeny, B. M., 2F

Thai, Theresa, 3G

Theelen, Thomas, 11

Tom, Manu, 30

Tong, Yubing, $\mathrm{OZ}$

Trésallet, Christophe, 2M

Treviño, Victor, 3C

Tseng, Tzu-Liang (Bill), 22

Tsuchida, T., 1N, 2O, 2W

Tsujimoto, Masakazu, 2V

Turkbey, Baris, OS

Turkbey, Evrim, 13, 1R

Uchiyama, Yoshikazu, $2 \mathrm{G}$

Udupa, Jayaram K., $\mathrm{OZ}$

Uranga, Javier, OY

Uthoff, J., 2T

van de Giessen, Martijn, 14

van de Leemput, Sil, 2P

van den Heuvel, T. L. A., $2 F$

van der Eerden, A. W., $2 F$ 
van Ginneken, Bram, 09, 11, 10 van Grinsven, Mark J. J. P., 11 van Oterendorp, Christian, 30 van Riel, Sarah J., 09, 10 van Rikxoort, Eva M., 10 van Uden, Inge, 11

Velthuis, Birgitta K., 12

Venhuizen, Freerk G., 11

Verdonschot, Nico, 3A

Viergever, Max A., OD, 12

Vilaça, João L., 2E, 3H

Vliegenthart, Rozemarijn, OD

Vos, Pieter C., 12

Wainer, Jacques, 28

Wang, Huafeng, $1 \mathrm{~F}$

Wang, Jui-Kai, OF

Wang, Luyao, 3F

Wang, Shijun, OS

Watanabe, Osamu, OP

Wei, Jun, 08, OE, OK, 18, 1H

Weinstein, Susan P., 00, 12

Weisenthal, Samuel, OS

Wielingen, Geoffrey V. F., OD

Wiemker, Rafael, OB

Wilms, Matthias, 2B

Wilson, Mary, OL, 17

Windridge, D., $2 \mathrm{~L}$

Winkler Wille, Mathilde Marie, 09, 10

Wittenberg, Thomas, 31

Wolf, Lior, OV

Wolford, Larry, 05

Wolterink, Jelmer M., OD

Wong, Ken C. L., 34

Wong, S. T., 31

Wood, Bradford J., OS

Wu, Jia, 00, 12

Wu, Peng, 35

Wu, Shandong, OM

Wu, Xia, 2 I

Xiaotao, Thomas, $1 \mathrm{~K}$

Xie, Qingguo, 3F

Xie, Yiting, 03, 0G, $1 G$

$\mathrm{Xu}$, Fang, 35

$\mathrm{Xu}$, Lele, $2 \mathrm{I}$

$\mathrm{Xu}, \mathrm{TaO}, \mathrm{XX}$

Yabuuchi, Hidetake, IW

Yaguchi, Atsushi, 2S

Yamagata, Hitoshi, $2 S$

Yamamuro, Osamu, 2A, 2V

Yan, Hong-jian, 35

Yang, Qing, $1 X$

Yang, Xiaofeng, 35

Yankelevitz, David F., OG, OI, IG

Yao, Jianhua, 13, 34, 3D

Yao, Li, 2

Yokoyama, Ryujiro, 3K

Yoshida, Hiroyuki, OQ, 2 Y

Yu, Ning, 00, $1 \mathrm{Z}$

Yuan, Rong, 3F

Zeng, Rongping, OC, IV
Zhang, Guopeng, 30

Zhang, Jianying, 22

Zhang, $\mathrm{Xi}, 3 \mathrm{O}$

Zhao, Yang, $1 \mathrm{~F}$

Zheng, Bin, 16, 1M, 20, 22, 24, 29, 3G

Zhong, L., 31, 32

Zhou, Chuan, 08, 0E, 1H, 1Q, 1S

Zhou, J., OL

Zhou, MU, 15, 1U, 2H, 2K

Zhou, Qin-Wu, 35

Zhou, Xiangrong, 33, 3K

Zhou, Xinxin, 3K

Zimmerman-Moreno, Gali, 1P

Zoghbi, Jihan M., 38

Zuley, Margarita L., OM 
Proc. of SPIE Vol. $9414941401-18$

Downloaded From: https://www.spiedigitallibrary.org/conference-proceedings-of-spie on 26 Apr 2023 Terms of Use: https://www.spiedigitallibrary.org/terms-of-use 


\title{
Conference Committee
}

\author{
Symposium Chairs
}

David Manning, Lancaster University (United Kingdom)

Steven C. Horii, The University of Pennsylvania Health System

(United States)

Conference Chairs

Lubomir M. Hadjiiski, University of Michigan Health System

(United States)

Georgia D. Tourassi, Oak Ridge National Laboratory (United States)

Conference Program Committee

Samuel G. Armato III, The University of Chicago (United States)

Susan M. Astley, The University of Manchester (United Kingdom)

Stephen Aylward, Kitware, Inc. (United States)

Kyongtae Ty Bae, University of Pittsburgh Medical Center

(United States)

Matthew S. Brown, University of California, Los Angeles (United States)

Heang-Ping Chan, University of Michigan Health System

(United States)

Marleen de Bruijne, Erasmus MC (Netherlands)

Thomas M. Deserno, RWTH Aachen (Germany)

Catalin Fetita, Télécom SudParis (France)

Hiroshi Fujita, Gifu University School of Medicine (Japan)

Maryellen L. Giger, The University of Chicago (United States)

Hayit Greenspan, Tel Aviv University (Israel)

Horst Karl Hahn, Fraunhofer MEVIS (Germany)

Khan M. Iftekharuddin, Old Dominion University (United States)

Nico Karssemeijer, Radboud University Nijmegen Medical Centre (Netherlands)

JongHyo Kim, Seoul National University Hospital (Korea, Republic of)

Joseph Y. Lo, Duke University Medical Center (United States)

Marius George Linguraru, Children's National Medical Center

(United States)

Kensaku Mori, Nagoya University (Japan)

Janne J. Näppi, Massachusetts General Hospital (United States)

Meindert Niemeijer, IDx, LLC (United States)

Noboru Niki, University of Tokushima (Japan)

Carol L. Novak, Siemens Corporation, Corporate Technology

(United States) 
Nicholas A. Petrick, United States Food and Drug Administration (United States)

Clarisa I. Sánchez, Radboud University Nijmegen Medical Centre (Netherlands)

Ronald M. Summers, National Institutes of Health (United States)

Kenji Suzuki, Illinois Institute of Technology (United States)

Bram van Ginneken, Radboud University Nijmegen Medical Centre (Netherlands)

Eva M. van Rikxoort, Radboud University Nijmegen Medical Centre (Netherlands)

Rafael Wiemker, Philips Research (Germany)

Axel Wismüller, University of Rochester Medical Center (United States)

Xiaofeng Yang, Emory University (United States)

Hiroyuki Yoshida, Massachusetts General Hospital (United States)

\section{Session Chairs}

Musculoskeletal and Miscellaneous

Axel Wismüller, University of Rochester Medical Center (United States)

Rafael Wiemker, Philips Research (Germany)

Lung and Chest I

Matthew S. Brown, University of California, Los Angeles (United States)

Catalin Fetita, Télécom SudParis (France)

Vessels, Heart and Eye I

Heang-Ping Chan, University of Michigan Health System (United States)

Stephen Aylward, Kitware, Inc. (United States)

Breast I

Joseph Y. Lo, Duke University School of Medicine (United States)

Hiroshi Fujita, Gifu University School of Medicine (Japan)

Prostate and Colon I

Ronald M. Summers, National Institutes of Health (United States)

Hiroyuki Yoshida, Massachusetts General Hospital (United States)

Keynote and Novel Methods

Kenji Suzuki, Illinois Institute of Technology (United States)

Horst K. Hahn, Fraunhofer MEVIS (Germany)

Head and Neck

Hayit Greenspan, Tel Aviv University (Israel)

Khan M. Iftekharuddin, Old Dominion University (United States) 
Breast II

Maryellen L. Giger, The University of Chicago (United States)

Susan M. Astley, The University of Manchester (United Kingdom)

Prostate and Colon II

Kensaku Mori, Nagoya University (Japan)

Janne J. Näppi, Massachusetts General Hospital (United States)

Vessels, Heart, and Eye II

Thomas M. Deserno, University Hospital Aachen (Germany)

Marleen de Bruijne, Erasmus MC (Netherlands)

Lung and Chest II

Nico Karssemeijer, Radboud University Nijmegen Medical Centre (Netherlands)

Samuel G. Armato III, The University of Chicago (United States)

Multi-Organ

Nicholas A. Petrick, U.S. Food and Drug Administration (United States)

Carol L. Novak, Siemens Corporation, Corporate Technology (United States) 
Proc. of SPIE Vol. $9414941401-22$

Downloaded From: https://www.spiedigitallibrary.org/conference-proceedings-of-spie on 26 Apr 2023 Terms of Use: https://www.spiedigitallibrary.org/terms-of-use 


\section{Introduction}

Following the success of previous years, the CAD Conference at the SPIE Medical Imaging Symposium 2015 again invited original papers on all aspects related to $C A D$, including: theory, overall system development, database construction, segmentation, feature extraction, classifier design, workstation design, and evaluation.

In 2015, the CAD Conference received 149 abstracts and offered acceptance to approximately $88 \%$. Abstract submissions came from various countries including: United States, Japan, Germany, the Netherlands, South Korea, Brazil, India, United Kingdom, Australia, Canada, France, Israel, Singapore, Spain, Portugal, China, and Mexico. The presentations spanned three-and-a-half days and included 64 oral presentations, 62 posters, a keynote address, a panel discussion, and a live demonstration workshop.

\section{Keynote}

On Monday morning, Dr. Tanveer Syeda-Mahmood from IBM Research - Almaden (United States) gave the CAD conference keynote on "Role of machine learning in clinical decision support". She was introduced by Dr. Larry Clarke from the National Cancer Institute ( $\mathrm{NCl}$ ), who discussed the importance of joint efforts among $\mathrm{NCl}$, academia, and industry for big data collection, storage, and analysis, machine learning, publicly available datasets, and Grand Challenges.

In the very inspiring keynote, Dr. Syeda-Mahmood presented the new IBM research avenues and initiatives related to clinical decision support. She discussed the role of advanced machine learning techniques and presented examples from practical multimodal decision support systems such as the IBM AALIM (Advanced Analytics for Information Management) system. The IBM AALIM system pioneered a new direction in evidence-based medicine using the concept of patient similarity and exploiting consensus opinions of other physicians who have seen similar patients. The fusion of healthcare data with inference algorithms from artificial intelligence, machine learning techniques, and patient similarity is driving a multimodal approach for clinical decision support.

In addition, Dr. Syeda-Mahmood discussed the IBM Medical Sieve Radiology Grand Challenge: a worldwide collaborative research effort across IBM research labs that is expanding patient data and knowledge-driven learning methods to define new clinical decision support systems for radiologists. The next generation for clinical decision support, she said, will involve cognitive assistants using multimodal reasoning and machine learning in all stages of disease detection. 


\section{LUNGx: the CAD Grand Challenge}

As part of the CAD Conference, SPIE, along with additional support from the American Association of Physicists in Medicine (AAPM) and the National Cancer Institute ( $\mathrm{NCl}$ ), conducted a "Grand Challenge" on developing quantitative image analysis methods for the diagnostic classification of malignant and benign lung nodules. This LUNGx Challenge provided a unique opportunity for participants to compare their algorithms to those of others from academia, industry, and government in a structured, direct way using the same data set.

The results of 13 algorithms from 11 groups were compared. An algorithm presented by Lyndsey Pickup, Mirada Medical UK (United Kingdom), was identified as the winner, and an algorithm presented by Yoganand Balagurunathan, Moffitt Cancer Center and University of Arizona (United States) was named runner-up. During the conference, participants presented posters about their LUNGx methods and results. The Challenge winner and runner-up were invited to join the special panel discussion. Additional information, including updates and lessons learned from the LUNGx Challenge, will be published soon in the SPIE Journal of Medical Imaging.

\section{Panel Discussion}

The special panel discussion on Tuesday afternoon focused on the topic of "CAD Grand Challenges-Present and Future." The moderators of the panel were Georgia D. Tourassi, Oak Ridge National Laboratory (United States) and Lubomir M. Hadjiiski, University of Michigan Health System (United States). The Panelists were: Samuel G. Armato, The University of Chicago (United States); Karen Drukker, The University of Chicago (United States); Laurence P. Clarke, National Cancer Institute (United States); George Redmond, National Cancer Institute (United States); Stephen Aylward, Kitware, Inc. (United States); Nicholas A. Petrick, U.S. Food and Drug Administration (United States); Lyndsey Pickup, Mirada Medical UK (United Kingdom), Yoganand Balagurunathan, Moffitt Cancer Center and University of Arizona (United States).

The panel provided a forum for discussion of the current experience with the LUNGX Challenge, other CAD grand challenges, and the future potential of these avenues for evaluation and testing of decision support systems. The panel included experts from academia, the National Cancer Institute (NCI) (United States), the Food and Drug Administration (FDA) (United States), industry, and the winners of the LUNGx Challenge. The panel members and audience discussed current and future opportunities for CAD Grand Challenges to become important testbeds and to enable cross platforms for decision support system evaluation. The panel agreed that by efficient planning and coordination among key organizing institutions, CAD Grand Challenges can play a vital role in the selection of promising classes of algorithms and systems for further clinical translational efforts and prompting advances in computer-aided diagnosis and ultimately precision medicine. 


\section{Live demonstration workshop}

In our annual Tuesday evening live demonstration workshop, 13 research groups interactively presented their real-time demonstrations of CAD workstations. The workshop was organized by Stephen Aylward, Kitware, Inc. (United States), and Heang-Ping Chan, University of Michigan Health System (United States). The applications ranged from detection, characterization, treatment monitoring, surgery, segmentation, and visualization aids in breast, lung, brain, abdomen, bladder, and spine imaging.

\section{Awards}

Ibrahim Sadek, Université de Bourgogne, (France) from our CAD Conference was named the Robert F. Wagner Best Student Paper First-Place Winner for the entire 2015 Medical Imaging Symposium, for his paper, "Automatic discrimination of color retinal images using the bag of words approach," [9414-54].

The high level of participation in all venues of the CAD conference gave a clear indication of the substantial active research work in the CAD field and the importance of the SPIE Medical Imaging CAD Conference as a major meeting place to present and discuss ongoing research and future directions.

Lubomir M. Hadjiiski

Georgia D. Tourassi 
Proc. of SPIE Vol. $9414941401-26$

Downloaded From: https://www.spiedigitallibrary.org/conference-proceedings-of-spie on 26 Apr 2023 Terms of Use: https://www.spiedigitallibrary.org/terms-of-use 


\section{Medical Imaging Award Recipients}

\section{Robert F. Wagner Award}

Robert F. Wagner was an active scientist in the SPIE Medical Imaging meeting, starting with the first meeting in 1972 and continuing throughout his career. He ensured that the BRH, and subsequently the $\mathrm{CDRH}$, was a sponsor for the early and subsequent Medical Imaging meetings, helping to launch and ensure the historical success of the meeting. The Robert $F$. Wagner All-Conference Best Student Paper Award (established 2014 ) is acknowledgment of his many important contributions to the Medical Imaging meeting and his many important advances to the field of medical imaging.

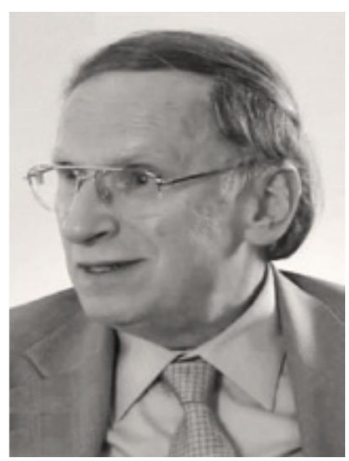

This award is cosponsored by:

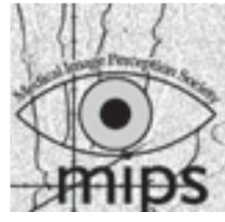

The Medical Image Perception Society

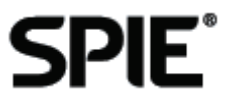

\section{Recipients:}

First Place: Automatic discrimination of color retinal images using the bag of words approach (9414-54)

I. Sadek, D. Sidibé, F. Meriaudeau, Univ. of Burgundy (France)

Second Place: Automated pulmonary lobar ventilation measurements using volumematched thoracic CT and MRI (9417-42)

F. Guo, S. Svenningsen, E. Bluemke, M. Rajchl, J. Yuan, A. Fenster, G. Parraga, The Univ. of Western Ontario (Canada)

\section{Conference Awards}

\section{Recipients:}

Cum Laude Poster Award: A new CAD approach for improving efficacy of cancer screening [9414-74]

B. Zheng, M. Tan, Y. Qiu, The Univ. of Oklahoma (United States); W. Qian, F. Lure, The Univ. of Texas at El Paso (United States); L. Li, Hangzhou Dianzi Univ. (China); J. Pu, Univ. of Pittsburgh (United States); Y. Kang, Northeastern Univ. (China) 
Proc. of SPIE Vol. $9414941401-28$

Downloaded From: https://www.spiedigitallibrary.org/conference-proceedings-of-spie on 26 Apr 2023 Terms of Use: https://www.spiedigitallibrary.org/terms-of-use 